eine Sauerstofftherapie, im Hinblick auf ihre Langzeitwirkung zu überprüfen.

Limitationen der Studie sind neben der geringen Erfolgsquote bei der CPAP-Anpassung sicherlich die fehlende Kontrollgruppe und die fehlende vollständige Polysomnografie.

\section{Fazit}

Die Studienergebnisse sind im Hinblick auf das Management von Schlaganfallpa- tienten für den klinischen Alltag von großer Bedeutung, denn sie zeigen, dass jeder Schlaganfallpatient unbedingt schlafmedizinisch untersucht werden sollte, um etwaige schlafbezogene Atmungsstörungen frühzeitig erkennen und eine entsprechende Therapie einleiten zu können. Diese wird dann die Prognose der ohnehin im Hinblick auf die Mortalität zur Hochrisikogruppe zählenden Patienten voraussichtlich verbessern können.

\section{Literatur}

1. Adams HP jr et al. Stroke 2007;38:1655-1711

2. Sacco RL et al. Circulation 2006;113:e409e449

3. Hankey GJ et al. Stroke 2000;31:2080-2086

4. Para O et al. Eur Respir J. 2004;24:267-272

5. Martínez-García MA et al. Chest 2005;128: 2123-2129

6. Sahlin et al. Arch Intern Med. 2008;168:297301

7. Turgington PM et al. Thorax 2004;59:367-371

\title{
ECMO-basiertes Therapiekonzept verbessert das Überleben
}

Peek GJ et al. Efficacy and economic assessment of conventional ventilatory support versus extracorporeal membrane oxygenation for severe adult respiratory failure

(CESAR): a multicentre randomised controlled trial. Lancet 2009;374:1351-1363

\section{Hintergrund}

Akutes Lungenversagen (acute respiratory distress syndrome, $\mathrm{ARDS}$ ) ist mit einer hohen Mortalität (ca. 30-60\%) und Morbidität assoziiert. Die Grundlage des multimodalen Therapiekonzepts in der Frühphase des ARDS bilden neben der ursächlichen Behandlung die Optimierung des Volumenstatus bzw. der Hämodynamik, die kinetische Therapie (intermittierende Bauchlage), der Einsatz inhalativer Vasodilatatoren (NO, Prostazykline) sowie eventuell die Gabe systemischer Steroide. Besondere Bedeutung kommt der lungenprotektiven Beatmung („low volume, low pressure“) und der Applikation einer möglichst niedrigen inspiratorischen Sauerstoffkonzentration zu. Um diese schonende Beatmung zu ermöglichen und die lebensbedrohliche Hypoxämie zu beseitigen, könnte die extrakorporale Membran-Oxygenierung (ECMO) eine wertvolle Therapieoption darstellen.

\section{Fragestellung}

Ältere randomisierte Studien, die die Effektivität dieser extrakorporalen Verfahren im Vergleich zur konventionellen
Therapie ohne ECMO untersuchten, erbrachten keine signifikanten Unterschiede im Überleben. Allerdings sind diese Studien unter Berücksichtigung der zwischenzeitlich veränderten Therapieund Beatmungsstrategien und der technischen Weiterentwicklungen wenig aufschlussreich. Deshalb wurden die Sicherheit, Effektivität und Kostenaspekte der ECMO in einer neuerlichen randomisierten, kontrollierten Studie (CESARStudie) evaluiert.

\section{Methodik}

Eingeschlossen in die Studie waren Patienten im Alter von 18 bis 65 Jahren und einem potenziell reversiblen respiratorischen Versagen mit einem MurrayScore von mindestens 3 und einem $\mathrm{pH}$ $<7,20$. Ausgeschlossen waren Patienten mit protrahiertem Lungenversagen ( 7 Tage) oder anderen Kontraindikationen für eine ECMO.

Die Randomisierung erfolgte 1:1 in die Kontrollgruppe mit konventioneller Positivdruckbeatmung bzw. Hochfrequenz-Oszillationsventilation (HFOV) oder in die Gruppe, die für eine Therapie mit (veno- venöser) ECMO in Betracht gezogen wurde. Diese erfolgte nur in einem ausgewählten spezialisierten Zentrum zusammen mit einem standardisierten Therapieregime.

Primärer Endpunkt war Tod oder schwere körperliche Behinderung/Einschränkung sechs Monate nach der Randomisierung.

\section{Ergebnisse}

Von Juli 2001 bis August 2006 wurden 180 Patienten randomisiert. Nur $68 \mathrm{~Pa}-$ tienten konnten einer ECMO zugeführt werden, da bei vielen Patienten bereits eine Optimierung der konventionellen Therapie erfolgreich war.

Patienten mit (möglicher) ECMOTherapie erhielten häufiger Steroide, eine Leberunterstützungstherapie (MARS) und eine lungenprotektive Beatmung. Bei Patienten, bei denen Informationen über den funktionellen Zustand bzw. über die Behinderung nach sechs Monaten vorlagen, war das Überleben ohne relevante Behinderung bei Patienten aus der ECMO-Gruppe knapp signifikant besser als in der Kontrollgruppe (63 vs. $47 \%$ ). 
Vorteile durch die ECMO ergaben sich auch hinsichtlich der Überlebenszeit (Abb. 1), nicht jedoch hinsichtlich der Überlebensrate. Die Aufenthaltsdauer in der Klinik bzw. auf der Intensivstation war in der Kontrollgruppe kürzer. Soweit erhoben ergaben sich keine signifikanten Unterschiede bezüglich Funktionsstatus, Lebensqualität oder Lungenfunktion. Die unmittelbaren Kosten waren in der ECMO-Gruppe zwar mehr als doppelt so hoch, die Kosten-Nutzen-Analyse unter Berücksichtigung der qualitätsadjustierten Lebensjahre (QALY) erbrachte aber Vorteile zugunsten der ECMOGruppe.

\section{Schlussfolgerung}

Bei Patienten mit akutem Lungenversagen kann durch ein ECMO-basiertes Therapieregime ein besseres Überleben ohne bleibende Schäden bei gleichzeitig höherer Kosteneffizienz erreicht werden.

\section{Kommentar}

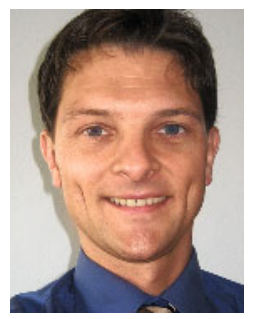

Priv.-Doz. Dr. med. Stephan Budweiser, Regensburg

Die Argumente für den Einsatz einer ECMO liegen im Wesentlichen darin begründet, dass durch die verbesserte Oxygenierung nicht nur mehr Zeit für Diagnostik und Therapie gewonnen wird, sondern auch, dass durch die dann mögliche Reduktion der Beatmungsinvasivität die Wahrscheinlichkeit für einen beatmungsassoziierten Lungenschaden vermindert wird. Dabei sind allerdings auch die Risiken des Verfahrens, (z.B. Gefäßkomplikationen, Ischämie, Blutungsrisiko, technische Probleme) und die Tatsache, dass viele Patienten mit ARDS aus verschiedenen Gründen (u.a. Gerinnungsversagen) dafür nicht geeignet sind, zu berücksichtigen. Dementsprechend musste auch in der vorliegenden Studie ein relativ hoher Prozentsatz von 76,5\% der initial in Betracht gezogenen Patienten primär ausgeschlossen werden. Häufige Gründe dafür sind - wie in dieser Untersuchung beschränkte Kapazitäten der Intensivme-

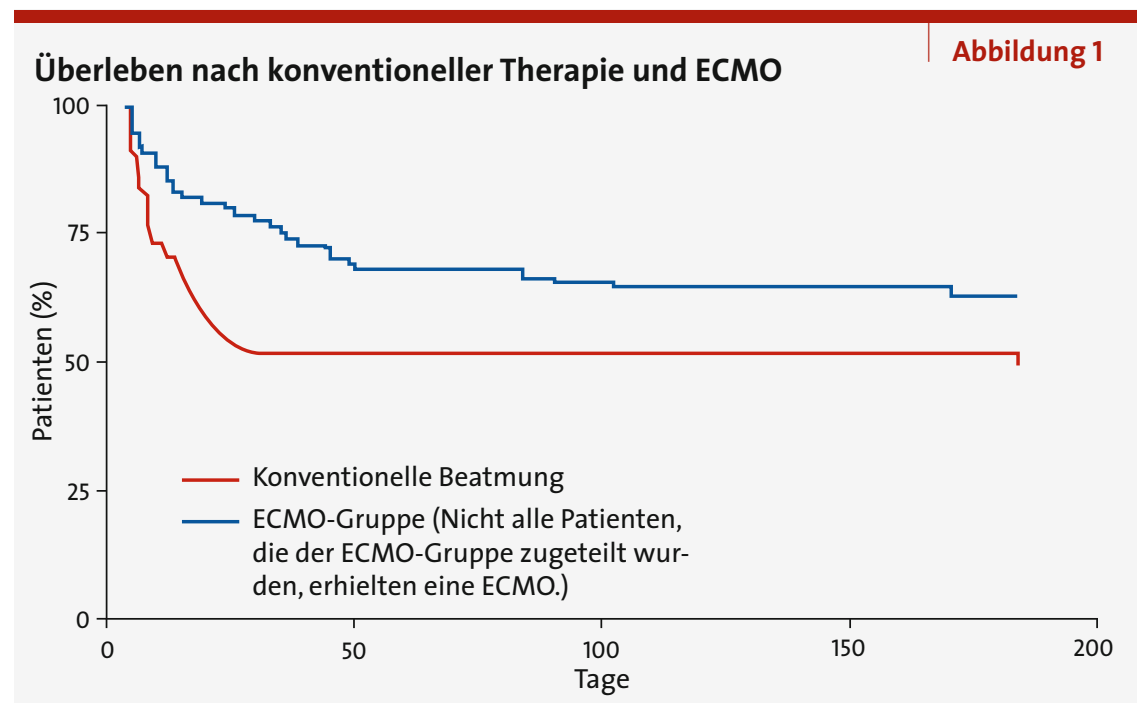

dizin bzw. ein bereits protrahierter Krankheitsverlauf.

Diese Studie, die mit hohem logistischem, apparativem und personellem Aufwand durchgeführt wurde, bringt einige wissenschaftliche Evidenz dafür, dass der Einsatz der ECMO zusammen mit einem standardisierten Therapiekonzept den Behandlungserfolg bei Patienten mit schweren Lungenschäden signifikant verbessern kann. Interessanterweise ergab sich rechnerisch trotz des primär hohen finanziellen Aufwands unter Berücksichtigung der QALY langfristig eine günstige Kosten-Nutzen-Relation.

Trotz der Euphorie für dieses sehr aufwendige Therapieverfahren ist jedoch zu erwähnen, dass sich die Ergebnisse sehr nahe am Signifikanzniveau bewegen und nur für den kumulativen Endpunkt, nicht jedoch für den Endpunkt Tod allein signifikant waren.

Zudem muss eingeräumt werden, dass diese Untersuchung keinen definitiven Aufschluss über den tatsächlichen Nutzen der ECMO per se für Patienten mit schwerem respiratorischem Versagen zulässt, da die ECMO-Therapie nur ein Bestandteil eines umfassenden Therapieregimes eines einzigen Zentrums mit hoher Expertise war. Dementsprechend konnte in diesem Zentrum bei etwa einem Fünftel der Patienten durch eine alleinige Optimierung der konventionellen Therapie einschließlich Beatmung eine signifikante Verbesserung auch ohne ECMO erreicht werden. Demgegenüber gab es für die Patienten der Kontrollgruppe, die über mehrere Zentren verteilt waren, außer der Vorgabe der lungenprotektiven Beatmung keine weitere Standardisierung der Therapie. Auch diese Studie deutet darauf hin, dass das Risiko des Transports dieser Patienten nicht unerheblich ist und stets gegenüber dem potenziellen Nutzen abgewogen werden muss.

\section{Fazit}

Die Daten sprechen dafür, dass bei Vorliegen dieses höchst lebensbedrohlichen Krankheitsbilds eine rechtzeitige Kontaktaufnahme mit einem Zentrum mit hoher Erfahrung im Umgang mit ARDS-Patienten und speziell mit extrakorporalen Behandlungsverfahren aufgenommen werden sollte, um geeigneten Patienten die optimale Therapie zukommen zu lassen.

Hinsichtlich extrakorporaler Verfahren können zukünftig bei suffizienten Kreislaufverhältnissen ohne unmittelbar lebensbedrohliche Hypoxämie vielleicht auch alternative, weniger aufwendige und bisweilen auch kostengünstigere Therapieoptionen wie etwa die pumpenlose arteriovenöse Lungenunterstützung (interventional Lung Assist, iLA) zum Einsatz kommen $[1,2]$. Die Effektivität dieses Verfahrens wird derzeit in einer prospektiven deutschen Multicenterstudie überprüft.

\section{Literatur}

1. Bein Th et al. A new pumpless extracorporeal interventional lung assist in critical hypoxemia/hypercapnia. Crit Care Med. 2006;34:1372-1377

2. Florchinger B et al. Pumpless extracorporeal lung assist: a ten year institutional experience. Ann Thorac Surg. 2008;86:410-417 